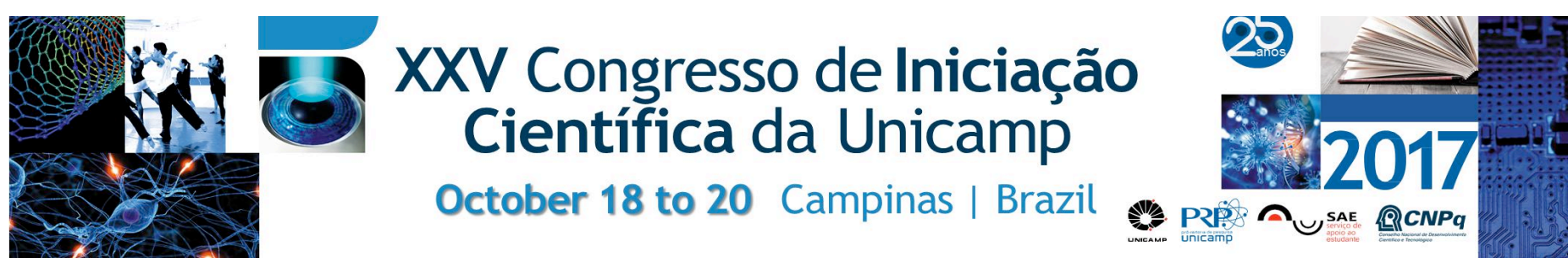

\title{
Molecular study of genes associated with steroid-resistant nephrotic syndrome
}

João Victor Virgilio-da-Silva*; Liliane Prates; Anna Cristina Gervásio de Britto Lutaif; Andréa Trevas MacielGuerra; Vera Maria Santoro Belangero; Gil Guerra-Júnior; Maricilda Palandi de Mello; Mara Sanches Guaragna

\begin{abstract}
Nephrotic syndrome (NS) is defined by heavy proteinuria, hypoalbuminemia, edema and hyperlipidemia. Recent advances in molecular genetics have identified single-gene causes of SRNS in more than 50 genes that encodes for structures of the glomerular filtration barrier (GFB). The aim of this study was the analysis of three variants previously identified by our group by whole exome sequencing (WES): the screening of c.459C>G (ACTN4) in 144 healthy controls and the validation by Sanger sequencing of the c.883_885dupTCT (ANLN) and c.3322A $>C$ (LAMB2) variants.
\end{abstract}

\section{Key words:}

Steroid resistant nephrotic syndrome; variants; ACTN4, LAMB2, ANLN genes.

\section{Introduction}

Nephrotic syndrome (NS), one of the most common kidney conditions in childhood, is characterized by massive proteinuria, hypoalbuminemia, edema and hyperlipidemia. Approximateley $20 \%$ of the children do not respond well to corticosteroid treatment and are classified as steroid-resistant NS (SRNS) ${ }^{1}$. Recent advances in molecular genetics have identified singlegene causes of SRNS in more than 50 genes that encodes for the glomerular filtration barrier (GFB) ${ }^{2}$.

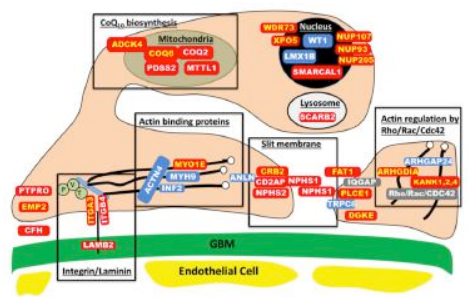

Figure 1: Glomerular filtration barrier. The glomerular filtration barrier complex. Podocyte foot processes are shown in light beige. GBM = Glomerular filtration barrier ${ }^{2}$.

Recently our group identified three novel heterozygous variants by WES in three different genes (Table 1). Therefore, the aims of this study were: 1) the screening of the ACTN4 variant in approximately 150 controls; 2) the validation by Sanger sequencing of the $A N L N$ and $L A M B 2$ variants.

Table 1: Variants analyzed in this study

\begin{tabular}{ccccc}
\hline Patients & Variant & Gene & ${\text { AD } / A^{a}}^{a}$ & Analysis \\
\hline P1 & p.Phe153Leu & ACTN4 & AD & Controls \\
\hline P2 & p.Ser295dup & $A N L N$ & $\mathrm{AD}$ & Sanger \\
\hline P3 & p.Asn1108Hys & LAMB2 & AR & Sanger \\
\hline
\end{tabular}

${ }^{a} \mathrm{AD}=$ autosomal dominant; $\mathrm{AR}=$ autosomal recessive.

\section{Results and Discussion}

The DNA was extracted by phenol:choloroform standar methods. Further PCR amplification using specific oligonucleotides and direct sequencing by Sanger were performed. Figure 2 and 3 ilustrates the results.

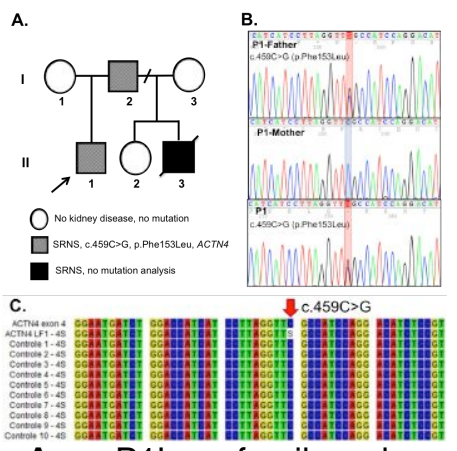

Figure 2: A. P1's family heredogram. B. Electropherograms (Chromas 2.6.2) of ACTN4 variant validation. C. CLC sequence viewer alignment showing the $\mathrm{C}>\mathrm{G}$ position. Only 10 controls are showed.

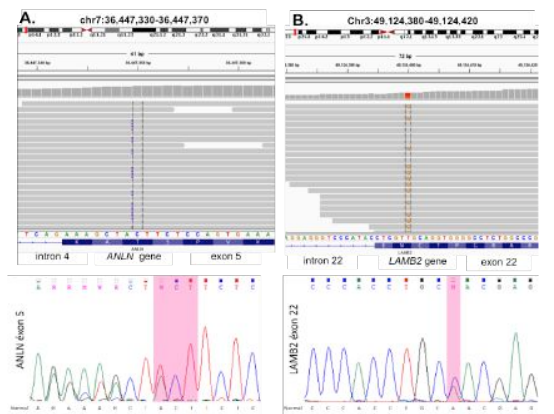

Figure 3: $A N L N(\mathbf{A})$ and $L A M B(B)$ variants identified by WES and analyzed by the IGV - 2.3 .68 (above) and respective electropherogams (Chromas 2.6.2) (below).

\section{Conclusions}

The variant identified in ACTN4 gene was not identified $(0 \%)$ in the 144 healthy controls screened and should be considered for further functional studies. The two variants identified in $A N L N$ and $L A M B 2$ genes were validated and should also be screened in controls.

\section{Acknowledgement} CBMEG, FAPESP, CNPq e SAE

${ }^{1}$ Benoit G, Machuca E, Antignac C. Hereditary nephrotic syndrome: a systematic approach for genetic testing and a review of associated podocyte gene mutations. Pediatr Nephrol. 2010;25(9):1621-32.

${ }^{2}$ Lovric S, Ashraf S, Tan, W, Hildebrandt, F. Genetic testing in steroid resistant

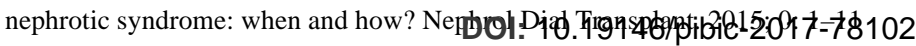

